\title{
AN EXPONENTIALLY-SMOOTHED GRAM-TYPE FORMULA FOR THE RIEMANN ZETA FUNCTION
}

\author{
R. B. Paris and S. Cang
}

\begin{abstract}
We examine the asymptotic nature of an expansion for $\zeta(s)$ where $s=\sigma+i t$, in which the terms of the original Dirichlet series (valid in $\sigma>1$ ) are smoothed by an exponential factor. It is found that in the critical strip $0<\sigma<1$ when $|t| \gg 1$, the "cut-off" in this smoothed sum occurs after $O(|t|)$ terms, so that the expansion can be said to be of Gram type. Numerical examples are given to illustrate the accuracy of the expansion.
\end{abstract}

\section{Introduction}

The earliest published method of computing the Riemann zeta function $\zeta(s)$ as a function of the complex variable $s=\sigma+i t$ is the so-called Gram formula [4,5]. This results from an application of the Euler-Maclaurin summation formula and is an asymptotic expansion valid for all $s(\neq 1)$ given by

$$
\zeta(s) \sim \sum_{n=1}^{N-1} n^{-s}+\frac{N^{1-s}}{s-1}+\frac{1}{2} N^{-s}+N^{-s} \sum_{m=1}^{\infty} \frac{B_{2 m}(s)_{2 m-1}}{(2 m) ! N^{2 m-1}}
$$

where $B_{2 m}$ are the Bernoulli numbers and $(\alpha)_{m}=\Gamma(\alpha+m) / \Gamma(\alpha)$. The asymptotic parameter in this expansion is $N$, and inspection of the terms in the sum over $m$ shows that for this to possess an asymptotic character (i.e., for the absolute value of the terms to initially decrease before ultimately diverging), $N$ must be chosen such that $N>|s| / 2 \pi$. On the critical line $\sigma=\frac{1}{2}$, the number of terms in the finite main sum (over $n$ ) in (1.1) when $t$ is large is thus of $O(|t|)$.

A more powerful means of computing $\zeta(s)$ on the critical line for large $t(>0)$ is the Riemann-Siegel formula [3,5]. This is usually presented for the real, even function $Z(t)=\exp (i \vartheta(t)) \zeta\left(\frac{1}{2}+i t\right)$ where the phase-angle $\vartheta(t)=\arg \Gamma\left(\frac{1}{4}+\frac{1}{2} i t\right)-\frac{1}{2} t \log \pi$. This (probably) asymptotic formula is given by

$$
Z(t) \sim 2 \operatorname{Re} e^{i \vartheta} \sum_{n=1}^{N_{t}} n^{-\frac{1}{2}-i t}+(-)^{N_{t}-1}(t / 2 \pi)^{-\frac{1}{4}} \sum_{r=0}^{\infty}(-)^{r}(t / 2 \pi)^{-\frac{1}{2} r} \Psi_{r}(z)
$$

where $N_{t}$ denotes the integer part of $(t / 2 \pi)^{\frac{1}{2}}, z=2\left\{(t / 2 \pi)^{\frac{1}{2}}-N_{t}\right\}-1$, and the functions $\Psi_{r}(z)$ are combinations of derivatives of the function $\Psi_{0}(z)=$ $\cos \pi\left(\frac{1}{2} z^{2}+\frac{3}{8}\right) / \cos \pi z$. For $t \gg 1$, this formula is a more efficient means of computing $\zeta\left(\frac{1}{2}+i t\right)$ since the main sum involves $N_{t} \simeq(t / 2 \pi)^{\frac{1}{2}}$ terms as compared with $O(t)$ terms in the Gram formula.

Recently, some new asymptotic formulas for $Z(t)$ have been developed in which the terms in the original Dirichlet series $\sum_{n=1}^{\infty} n^{-s}$ are smoothed by a function which 
is approximately unity for $n \lesssim N_{t}$ and decays to zero for $n \gtrsim N_{t}$. The first is an expansion derived by Berry and Keating [2], in which the leading term is given by the convergent sum

$$
2 \operatorname{Re} e^{i \vartheta} \sum_{n=1}^{\infty} n^{-\frac{1}{2}-i t} \times \frac{1}{2} \operatorname{erfc}\left\{\left(\frac{t}{2}\right)^{\frac{1}{2}} \frac{\xi(n, t)}{q(\kappa, t)}\right\}
$$

where $\xi(n, t)=\log n-\vartheta^{\prime}(t), q^{2}(\kappa, t)=\kappa^{2}-i t \vartheta^{\prime \prime}(t)$, and $\kappa$ is a real free parameter. For large $t, \xi(n, t) \simeq \log \left(n / N_{t}\right)$ and $q^{2}(\kappa, t) \simeq \kappa^{2}-\frac{1}{2} i$, so that (1.3) resembles the finite main sum of the Riemann-Siegel formula, but with the sharp cut-off after $N_{t}$ terms smoothed away by the complementary error function. One advantage of such a smoothed formula over the Riemann-Siegel formula is the removal of the discontinuity in the main sum as a function of $t$ resulting from the discrete upper limit $N_{t}$. Other expansions with the normalized incomplete gamma function $Q(a, z)=\Gamma(a, z) / \Gamma(a)$ as the smoothing function have been developed in [6,8]. In [8], an expansion for $Z(t)$ is obtained from the result

$$
Z(t)=2 \operatorname{Re}\left\{e^{i \vartheta} \sum_{n=1}^{\infty} n^{-s} Q\left(\frac{1}{2} s, \pi n^{2} i\right)-\frac{\pi^{\frac{1}{4}} e^{\frac{1}{4} \pi i s}}{s\left|\Gamma\left(\frac{1}{2} s\right)\right|}\right\}
$$

on the critical line $s=\frac{1}{2}+i t$. The uniform asymptotics of the incomplete gamma function for large $t$ shows that it can be represented in terms of a complementary error function, with the result that $Q\left(\frac{1}{2} s, \pi n^{2} i\right)$ is approximately unity for $n \lesssim N_{t}$ and decays to zero for $n \gtrsim N_{t}$. Thus, the expansion (1.4) also involves a main sum smoothed by a complementary error function, although with a different argument to that in (1.3). Since the cut-off occurs after roughly $N_{t}$ terms in both the expansions (1.3) and (1.4), we see that these formulas are both of the computationally more powerful Riemann-Siegel type.

In this paper, we derive and investigate a generalisation of the expansion (1.4), which involves the incomplete gamma functions with a free parameter $a$, instead of $\frac{1}{2} s$. Suitable choice of this parameter then enables us to present expansions in which the main sum is smoothed either by an exponential factor or a complementary error function of real argument. Examination of the asymptotics of the correction terms and the remainder term in these expansions shows, however, that for these terms to possess an asymptotic character, we are forced to take the cut-off in the smoothed main sum to occur after $O(t)$ terms. Consequently, it seems the price to be paid for such simple smoothing of the Dirichlet series is that the resulting formulas are of the less powerful Gram type.

\section{A generalised expansion for $\zeta(s)$}

Let $p$ be a positive real number and $a, K$ denote arbitrary complex parameters which will be restricted to satisfy $|\arg K|<\pi / 4 p$ and, for convenience, $\operatorname{Re}(a) \geq 0$. We employ the Mellin integral representation of the incomplete gamma function $\Gamma(a, x)$ given by

$$
\Gamma(a, x)=\frac{1}{2 \pi i} \int_{c-\infty i}^{c+\infty i} \Gamma(a+u) x^{-u} \frac{d u}{u}, \quad|\arg x|<\frac{1}{2} \pi
$$


where $c>0$, to express the absolutely convergent sum (valid for all values of $s$ ) of incomplete gamma functions in the form

$$
\begin{gathered}
\sum_{n=1}^{\infty} n^{-s} Q\left(a,(n / K)^{2 p}\right)=\frac{1}{2 \pi i \Gamma(a)} \int_{c-\infty i}^{c+\infty i} \Gamma\left(a+\frac{z}{2 p}\right) K^{z} \zeta(s+z) \frac{d z}{z} \\
|\arg K|<\pi / 4 p, \quad c>\max \{0,1-\sigma\} .
\end{gathered}
$$

This Perron-type formula is readily established by reversal of the order of summation and integration (justified by absolute convergence) followed by introduction of the new variable $z=2 p u$.

The integrand in (2.1) has poles at $z=0$ and $z=1-s$. As we are primarily interested in $s$ values situated in the critical strip $0<\sigma<1$, we shall suppose for convenience in presentation that $\sigma \leq 1$. This ensures that the pole at $z=1-s$ lies in $\operatorname{Re}(z) \geq 0$, thereby avoiding the possibility of the formation of a double pole. Displacement of the path of integration and evaluation of the residues at the simple poles $z=0$ and $z=1-s$ then shows that

$$
\zeta(s)=\sum_{n=1}^{\infty} n^{-s} Q\left(a,(n / K)^{2 p}\right)+\frac{\Gamma\left(a+\frac{1-s}{2 p}\right)}{\Gamma(a)} \frac{K^{1-s}}{s-1}+J
$$

where

$$
J=\frac{i}{2 \pi \Gamma(a)} \int_{-c-\infty i}^{-c+\infty i} \Gamma\left(a+\frac{z}{2 p}\right) K^{z} \zeta(s+z) \frac{d z}{z},
$$

and $0<c / 2 p<\operatorname{Re}(a)$. We remark that the restriction on $\sigma$ is easily removed to include the case $\sigma>1$ by suitable indentation (when necessary) of the path of integration in (2.3) to lie to the left of the pole $z=1-s$ but to the right of the poles of the gamma function. ${ }^{1}$ This result also can be obtained by a generalization of the method described in [7] and application of the Poisson summation formula.

Straightforward displacement of the path of integration in (2.3) to the left over the simple poles of the gamma function, combined with the functional relation $\zeta(s)=$ $\chi(s) \zeta(1-s)$ where

$$
\chi(s)=2^{s} \pi^{s-1} \sin \frac{1}{2} \pi s \Gamma(1-s)=\pi^{s-\frac{1}{2}} \frac{\Gamma\left(\frac{1}{2}-\frac{1}{2} s\right)}{\Gamma\left(\frac{1}{2} s\right)},
$$

then leads to the result

$$
\begin{aligned}
J & =\frac{1}{\Gamma(a)} \sum_{m=0}^{M-1} \frac{(-)^{m}}{m !} \frac{K^{-2 p(m+a)}}{m+a} \zeta(s-2 p(m+a))+R_{M} \\
& =\frac{\chi(s)}{\Gamma(a)} \sum_{m=0}^{M-1} \frac{(-)^{m}}{m !} \frac{(2 \pi K)^{-2 p(m+a)}}{m+a} A_{m}+R_{M}
\end{aligned}
$$

where $M=1,2, \ldots$. The coefficients $A_{m}$ are defined by

$$
A_{m}=\frac{\sin \frac{1}{2} \pi(s-2 p(m+a))}{\sin \frac{1}{2} \pi s} \frac{\Gamma(1-s+2 p(m+a))}{\Gamma(1-s)} \zeta(1-s+2 p(m+a)),
$$

\footnotetext{
${ }^{1}$ This separation of the poles will not be possible when $s=1+2 p(m+a), m=0,1,2, \ldots$, due to the presence of a double pole at $z=1-s$. In this case, the second term on the right-hand side of (2.2) would disappear. Since this situation only can arise for $\sigma>1$, we do not consider this further.
} 
and the remainder $R_{M}$, when $|\arg K|<\pi / 4$, is given by

$$
R_{M}=\frac{i}{2 \pi \Gamma(a)} \int_{-D_{M}-\infty i}^{-D_{M}+\infty i} \Gamma\left(a+\frac{z}{2 p}\right) K^{z} \zeta(s+z) \frac{d z}{z}, \quad M=1,2, \ldots,
$$

where $D_{M}=c+2 p(M-1+a)$ and $0<c<2 p$.

Combination of (2.2) and (2.5) then gives the desired expansion for $\zeta(s)$ in the form

$$
\begin{aligned}
\zeta(s)= & \sum_{n=1}^{\infty} n^{-s} Q\left(a,(n / K)^{2 p}\right)+\frac{\Gamma\left(a+\frac{1-s}{2 p}\right)}{\Gamma(a)} \frac{K^{1-s}}{s-1} \\
& +\frac{\chi(s)}{\Gamma(a)} \sum_{m=0}^{M-1} \frac{(-)^{m}}{m !} \frac{(2 \pi K)^{-2 p(m+a)}}{m+a} A_{m}+R_{M} .
\end{aligned}
$$

This representation of $\zeta(s)$ is seen to involve the original Dirichlet series smoothed by the incomplete gamma function. We remark that the coefficients $A_{m}$ in (2.6) enjoy the unusual property of involving the zeta function itself. The expansion (2.8) therefore will be of computational use only if $\operatorname{Re}(a)>\sigma / 2 p$, for then the zeta functions in (2.6) can be computed simply from the convergent Dirichlet series. This expansion generalizes the result in (1.4), which corresponds to the particular choice $a=\frac{1}{2} s$ and $p=1$; the connection between the two expansions is discussed in Appendix A.

\section{A bound on $R_{M}$ when $a>0$}

A simple bound on $R_{M}$ in the critical strip $0<\sigma<1$, when $p \geq 1$ and $a, K$ are positive real (we recall from $\S 2$ that for (2.8) to be meaningful we require $a>\sigma / 2 p$ ), can be obtained as follows. We choose $c=p$ in (2.7) and employ the functional relation for $\zeta(s)$. With $s=\sigma+i t(t>0)$ and the new variable $z=-\delta+i u, u=y-t$, where $\delta=2 p\left(M+a-\frac{1}{2}\right)$, we then have, for $M=1,2, \ldots$,

$$
\begin{aligned}
R_{M}=\frac{i}{2 \pi \Gamma(a)} \int_{-\delta-\infty i}^{-\delta+\infty i} \Gamma\left(a+\frac{z}{2 p}\right) \zeta(1-s-z) K^{z} \chi(s+z) \frac{d z}{z} \\
=\frac{\pi^{s-\frac{3}{2}}}{2 \Gamma(a)} \int_{-\infty}^{\infty} \Gamma\left(-M+\frac{1}{2}+\frac{i u}{2 p}\right)(\pi K)^{-\delta+i u} \zeta(1-\sigma+\delta-i y) \\
\times \frac{\Gamma\left(\frac{1-\sigma+\delta}{2}-\frac{i y}{2}\right)}{\Gamma\left(\frac{\sigma-\delta}{2}+\frac{i y}{2}\right)} \frac{d y}{(\delta-i u)}
\end{aligned}
$$

From the result

$$
\left|\Gamma\left(\frac{1}{2}+\frac{i u}{2 p}\right)\right|=\left(\frac{\pi}{\cosh \pi u / 2 p}\right)^{\frac{1}{2}}<(2 \pi)^{\frac{1}{2}} e^{-\pi|u| / 4 p},
$$

it follows that

$$
\begin{aligned}
\left|\Gamma\left(-M+\frac{1}{2}+\frac{i u}{2 p}\right)\right| & =\left|\Gamma\left(\frac{1}{2}+\frac{i u}{2 p}\right)\right| \prod_{r=0}^{M-1}\left|\frac{1}{2}+r-\frac{i u}{2 p}\right|^{-1} \\
& <(2 \pi)^{\frac{1}{2}} e^{-\pi|u| / 4 p} \frac{\Gamma\left(\frac{1}{2}\right)}{\Gamma\left(M+\frac{1}{2}\right)}
\end{aligned}
$$


Use of the inequality $|\zeta(x+i y)| \leq \zeta(x)$ for real $x(>1)$ and $y$ then shows that for $0<\sigma<1$ (so that $1-\sigma+\delta>1$ )

$$
\left|R_{M}\right|<\frac{2^{-\frac{1}{2}} \pi^{\sigma-\frac{1}{2}}}{\Gamma(a)} \frac{(\pi K)^{-\delta}}{\delta \Gamma\left(M+\frac{1}{2}\right)} \zeta(1-\sigma+\delta) \int_{-\infty}^{\infty} e^{-\pi|u| / 4 p} F(y) d y
$$

where

$$
F(y)=\left|\frac{\Gamma\left(\alpha-\frac{1}{2} i y\right)}{\Gamma\left(\frac{1}{2}-\alpha+\frac{1}{2} i y\right)}\right|, \quad \alpha=\frac{1}{2}(1-\sigma+\delta) .
$$

In Appendix B, it is shown that $F(y)$ is an even, monotonically increasing function of $y$ when $\alpha>\frac{1}{4}$. From Stirling's formula, the scaled gamma function ratio $G(y)=$ $(y / 2)^{\frac{1}{2}-2 \alpha} F(y)$ is such that $G(y) \rightarrow 1$ as $y \rightarrow+\infty$, for fixed $\alpha$. This function also is discussed in Appendix B, where it is established that $G(y)$ is monotonically decreasing in $y$ for $y>0$ when $\alpha \geq 1$. Defining the integral in (3.1) by $I$ and using the above properties of $F(y)$, we can write

$$
\begin{aligned}
I & =\left\{\int_{t}^{\infty}+\int_{-t}^{t}+\int_{-\infty}^{-t}\right\} e^{-\pi|u| / 4 p} F(y) d y \\
& =2 \cosh \frac{\pi t}{4 p} \int_{t}^{\infty} e^{-\pi y / 4 p} F(y) d y+2 e^{-\pi t / 4 p} \int_{0}^{t} \cosh \frac{\pi y}{4 p} F(y) d y \\
& <2 \cosh \frac{\pi t}{4 p} G_{p}(M ; t) \int_{t}^{\infty} e^{-\pi y / 4 p}(y / 2)^{\frac{1}{2}-\sigma+\delta} d y+\frac{8 p}{\pi} e^{-\pi t / 4 p} \sinh \frac{\pi t}{4 p} F(t),
\end{aligned}
$$

since in the critical strip $\alpha=(1-\sigma+\delta) / 2>1$ for $p \geq 1, M=1,2 \ldots$, and $a>\sigma / 2 p$. To denote the dependence of the scaled gamma function ratio $G(y)$ on $p$ and $M$ (we omit the dependence on $\sigma$ and the parameter $a$ ), we have written

$$
G(y) \equiv G_{p}(M ; y)=(y / 2)^{\frac{1}{2}-2 \alpha} F(y) .
$$

The integral in (3.3) then can be evaluated as an incomplete gamma function to yield

$$
I<\frac{8 p}{\pi}\left(\frac{1}{2} t\right)^{N} C_{p}(M ; t), \quad N=2 \alpha-\frac{1}{2}
$$

where

$$
C_{p}(M ; t)=G_{p}(M ; t)\left\{e^{-X} \sinh X+X^{-N} \Gamma(N+1, X) \cosh X\right\}, \quad X=\pi t / 4 p .
$$

We note that the expression in braces in (3.6) tends to unity as $X \rightarrow \infty$ for fixed $N$. For $M$ and $t$ values such that $X>N(>0)$, use of the well-known bound for the incomplete gamma function, given by $X^{-N} \Gamma(N+1, X)<e^{-X} /(1-N / X)$, yields the simpler result

$$
C_{p}(M ; t)<G_{p}(M ; t)\left\{1+e^{-X} \cosh X /((X / N)-1)\right\}, \quad X>N .
$$

Hence, from (3.1), we finally obtain the bound on $R_{M}$ in the critical strip given by

$$
\left|R_{M}\right|<\frac{2^{\frac{3}{2}} \zeta(2 \alpha)}{\pi \Gamma(a)\left(M+a-\frac{1}{2}\right) \Gamma\left(M+\frac{1}{2}\right)}\left(\frac{t}{2 \pi}\right)^{\frac{1}{2}-\sigma}\left(\frac{t}{2 \pi K}\right)^{2 p\left(M+a-\frac{1}{2}\right)} C_{p}(M ; t)
$$

where we recall that $\alpha=\frac{1}{2}(1-\sigma)+p\left(M+a-\frac{1}{2}\right)$. The function $G_{p}(M ; t)$ is determined by direct computation from (3.2) and (3.4). In Table 1 we show values of $G_{p}(M ; t)$ for different $M$ when $a=p=1$ for $t=50$ and $t=100$. For low values of $M, G_{p}(M ; t) \simeq 1$ 
in accordance with the asymptotic approximation in (B.4). For larger values of $M$, it is found that $G_{p}(M ; t)$ ceases to be $O(1)$ once $M \gtrsim\left(\frac{1}{2} t\right)^{2 / 3} / p$.

\begin{tabular}{|c|c|c|}
\hline \multicolumn{3}{|c|}{$a=1 \quad p=1$} \\
\hline$M$ & $G_{p}(M ; 50)$ & $G_{p}(M ; 100)$ \\
\hline 5 & 1.09122 & 1.02230 \\
\hline 10 & 1.79844 & 1.16447 \\
\hline 15 & 5.99574 & 1.62045 \\
\hline 20 & 48.5599 & 2.98869 \\
\hline 25 & 1042.88 & 7.82060 \\
\hline
\end{tabular}

TABLE 1. Values of the coefficient $G_{p}(M ; t)$ when $a=1, p=1$ for $t=50$ and $t=100$.

\section{An exponentially-smoothed main sum}

In the remainder of this paper, we shall put $a=1$, since $Q(1, z)=e^{-z}$, and let $K$ be a real positive parameter. This choice results in the main sum in (2.8) being smoothed by the real exponential factor $\exp \left[-(n / K)^{2 p}\right]$. We note that the integral $(2.1)$ in this case is equivalent to that given in $[9, \S 9.43]$ for a general Dirichlet series.

We shall further suppose $p$ to be a positive integer (although this is not necessary), so that the coefficients $A_{m}$ in (2.6) assume the simpler form

$$
A_{m-1}=(-)^{p m}(1-s)_{2 p m} \zeta(1-s+2 p m), \quad m=1,2, \ldots
$$

Then, from (2.8) and (4.1), we obtain, for $p=1,2, \ldots$,

$$
\begin{aligned}
\zeta(s)= & \sum_{n=1}^{\infty} n^{-s} \exp \left[-(n / K)^{2 p}\right]-\frac{K^{1-s}}{2 p} \Gamma\left(\frac{1-s}{2 p}\right) \\
& -\chi(s) \sum_{m=1}^{M} \frac{(-)^{m}}{m !}(2 \pi K)^{-2 p m} A_{m-1}+R_{M}
\end{aligned}
$$

where $R_{M}$ is bounded by (3.8) in the critical strip $0<\sigma<1$.

The formula (4.2) exhibits an exponential smoothing of the terms in the infinite main sum (over $n$ ) which effectively "switch off" for values of $n$ given by $n^{*} \sim K$ where the parameter $K$, as yet, has not been specified. The choice of $K$, however, is unfortunately not entirely at our disposal. This results from the large $m$-behavior of the terms in the finite sum in (4.2) which, since $\zeta(1-s+2 p m) \rightarrow 1$ as $m \rightarrow \infty$, is controlled essentially by the behavior of

$$
a_{m} \equiv\left(\frac{t}{2 \pi K}\right)^{2 p m} \frac{(1-s)_{2 p m}}{m ! t^{2 p m}}, \quad m=1,2, \ldots
$$

It is easily shown that, for the terms in this sequence to possess an asymptotic character, it is necessary to choose $K \gtrsim t / 2 \pi$ (see also the bound on the remainder $R_{M}$ given in (3.8)). Since the main sum is smoothed after $n^{*} \simeq t / 2 \pi$ terms, the formula (4.2) has the character of a Gram-type formula, rather than that of the Riemann-Siegel-type formulas discussed in $[4,5,6]$. With $K$ chosen in this manner, the terms in (4.2) at 
first will decrease to a minimum value at $m=M_{0}$, before finally diverging in typical asymptotic fashion. The optimal truncation point $M_{0}$ is given approximately by

$$
M_{0} \simeq(t / 2 \lambda p)^{2 p /(2 p-1)}
$$

when $\lambda \equiv t / 2 \pi K=O(1)$.

The final parameter which is free to be chosen in (4.2) is $p$. First, it is seen that provided $p$ is chosen such that $t / 2 p \gg 1$, the finite series in (4.2) will possess an asymptotic character and thereby provide a means of accurately computing $\zeta(s)$ in the critical strip. Secondly, it is clear that increasing $p$ will be of computational advantage since (a) the terms in the smoothed main sum will decay more rapidly for $n>n^{*}$, and (b) the value of $\zeta(1-s+2 p m)$ will approach the limiting value unity more rapidly as $m$ increases and consequently will be easier to compute. However, this enhancement is offset by the fact that the sequence in (4.3) ultimately will lose its asymptotic character for fixed $t$ if $p$ becomes too large. In addition, the value of the minimum term of the asymptotic sum in (4.2) at optimal truncation will increase as $p$ increases, thereby limiting the attainable accuracy.

To illustrate the expansion in (4.2), we consider the critical line where, from (2.4), $\chi\left(\frac{1}{2}+i t\right)=\exp (-2 i \vartheta)$. We first take $p=1$ and choose $K=t / 2 \pi$. Then we have

$$
\begin{gathered}
\zeta(s)=\sum_{n=1}^{\infty} n^{-s} \exp \left[-(2 \pi n / t)^{2}\right]-\frac{1}{2}(t / 2 \pi)^{1-s} \Gamma\left(\frac{1-s}{2}\right) \\
-\chi(s) \sum_{m=1}^{M} \frac{(1-s)_{2 m}}{m ! t^{2 m}} \zeta(1-s+2 m)+R_{M}
\end{gathered}
$$

where $s=\frac{1}{2}+i t$. From (2.16), the remainder term satisfies the bound (when $\sigma=\frac{1}{2}$ )

$$
\left|R_{M}\right|<\frac{2^{\frac{3}{2}} \zeta\left(2 M+\frac{3}{2}\right) C_{1}(M ; t)}{\pi \Gamma\left(M+\frac{3}{2}\right)},
$$

where $\zeta\left(2 M+\frac{3}{2}\right) \simeq 1$ for $M \gg 1$ and the expression for $C_{1}(M ; t)$ is given in (3.6) (or (3.7)) with $X=\pi t / 4, N=2 M+1$.

In Table 2, we present the optimal truncation point $M_{0} \simeq t^{2} / 4$ for a range of $t$ values (see (4.4)), together with the absolute value of the least term in the finite series (over $m$ ) in (4.5) (see (4.3)). For the same $t$ values, Table 3 shows the real function $Z(t)$ computed from (4.5) with $M$ chosen to guarantee an accuracy of 25 decimal places. For the smallest value $t=10, M$ was chosen to be the optimal value $M_{0}=24$, while for the other $t$ values, truncation of the series (4.5) was highly sub-optimal.

\begin{tabular}{|c|l|l|}
\hline \multicolumn{3}{|c|}{$a=1 \quad p=1$} \\
\hline$t$ & $M_{0}$ & $\left|a_{M_{0}}\right|$ \\
\hline 10 & 24 & $2.711 \times 10^{-6}$ \\
20 & 99 & $2.405 \times 10^{-32}$ \\
30 & 224 & $5.496 \times 10^{-80}$ \\
40 & 400 & $2.725 \times 10^{-149}$ \\
50 & 625 & $2.780 \times 10^{-240}$ \\
\hline
\end{tabular}

TABLE 2. Optimal truncation values of the finite sum in (4.1) for different $t$ values when $a=p=1$. 


\begin{tabular}{|c|c|c|}
\hline \multicolumn{3}{|c|}{$a=1 \quad p=1$} \\
\hline$t$ & $Z_{\text {approx }}$ & $M$ \\
\hline 10 & -1.5491898595 & 24 \\
20 & +1.1478424121851972776350341 & 50 \\
30 & +0.5960285192398849553185143 & 33 \\
40 & -1.3088823934565991590161454 & 29 \\
50 & -0.3407350059550249827533166 & 27 \\
\hline
\end{tabular}

TABLE 3. Computation of $Z(t)$ from (4.1) for different values of $t$. When $t=10$, optimal truncation yields a value accurate to 5 decimals only. For the other $t$ values, $M$ was chosen to yield an accuracy of 25 decimal places.

\begin{tabular}{|l|l|l||l|l|}
\hline \multicolumn{5}{|c|}{$t=50 \quad M_{0}=625$} \\
\hline \multirow{3}{*}{$M$} & \multicolumn{2}{|c|}{$a=1$} & \multicolumn{2}{c|}{$a=1 / 2$} \\
\cline { 2 - 5 } & $\left|Z-Z_{\text {approx }}\right|$ & $\left|R_{M}\right|$ & $\left|Z-Z_{\text {approx }}\right|$ & $\left|R_{M}\right|$ \\
\hline 5 & $1.366 \times 10^{-3}$ & $4.047 \times 10^{-3}$ & $8.053 \times 10^{-4}$ & $2.284 \times 10^{-3}$ \\
10 & $4.588 \times 10^{-8}$ & $2.070 \times 10^{-7}$ & $2.411 \times 10^{-8}$ & $1.100 \times 10^{-7}$ \\
15 & $3.268 \times 10^{-13}$ & $2.464 \times 10^{-12}$ & $2.911 \times 10^{-14}$ & $1.156 \times 10^{-12}$ \\
20 & $1.217 \times 10^{-18}$ & $2.330 \times 10^{-17}$ & $1.273 \times 10^{-19}$ & $9.219 \times 10^{-18}$ \\
25 & $3.718 \times 10^{-24}$ & $5.198 \times 10^{-22}$ & $1.171 \times 10^{-24}$ & $1.608 \times 10^{-22}$ \\
30 & $1.188 \times 10^{-29}$ & $6.189 \times 10^{-26}$ & $4.643 \times 10^{-31}$ & $1.455 \times 10^{-26}$ \\
\hline
\end{tabular}

\begin{tabular}{|c|c|c|c|c|}
\hline \multicolumn{5}{|c|}{$t=100 \quad M_{0}=2500$} \\
\hline \multirow[b]{2}{*}{$M$} & \multicolumn{2}{|c|}{$a=1$} & \multicolumn{2}{|c|}{$a=1 / 2$} \\
\hline & $\left|Z-Z_{\text {approx }}\right|$ & $\left|R_{M}\right|$ & $\mid Z-Z_{\text {approx }}$ & $\mid R_{M}$ \\
\hline 5 & $1.250 \times 10^{-3}$ & $3.453 \times 10^{-3}$ & $6.461 \times 10^{-4}$ & $2.116 \times 10^{-3}$ \\
\hline 10 & $2.763 \times 10^{-8}$ & $1.038 \times 10^{-7}$ & $8.704 \times 10^{-9}$ & $5.967 \times 10^{-8}$ \\
\hline 15 & $7.709 \times 10^{-14}$ & $3.706 \times 10^{-13}$ & $1.064 \times 10^{-15}$ & $2.037 \times 10^{-13}$ \\
\hline 20 & $6.102 \times 10^{-20}$ & $3.689 \times 10^{-19}$ & $9.179 \times 10^{-21}$ & $1.945 \times 10^{-19}$ \\
\hline 25 & $2.123 \times 10^{-26}$ & $1.654 \times 10^{-25}$ & $1.078 \times 10^{-26}$ & $8.304 \times 10^{-26}$ \\
\hline 30 & $4.290 \times 10^{-33}$ & $4.605 \times 10^{-32}$ & $5.603 \times 10^{-34}$ & $2.184 \times 10^{-32}$ \\
\hline 40 & $6.996 \times 10^{-47}$ & $2.520 \times 10^{-45}$ & $9.583 \times 10^{-49}$ & $1.022 \times 10^{-45}$ \\
\hline
\end{tabular}

TABLE 4. Values of $\left|R_{M}\right|$ for $t=50$ and $t=100$ when $p=1, K=t / 2 \pi$.

The bound (4.6) is unfortunately not sharp enough to establish the asymptotic nature of the expansion in (4.5). For modest values of $M$ (which would, however, be quite sufficient for most computational purposes), this bound turns out to be very realistic, but is too crude for values of $M$ near optimal truncation. To illustrate this, in Table 4, we compare the bound (4.6) for different truncations $M$ with the actual error incurred in computing $Z(t)$ for two different values of $t$. Inspection of this table reveals that the bound is quite realistic until $M \simeq\left[\frac{1}{2} t\right]$. For higher values of $M,(4.6)$ begins to deteriorate and is quite useless at optimal truncation.

In Table 5, we present the results of computing $Z(t)$ from (4.5) with different values of $p$ and $K$ when $t=100$. This table illustrates two important facts. The first is that, although increasing $K$ clearly increases the attainable accuracy, this is at the 


\begin{tabular}{|c|l|c|}
\hline & \multicolumn{1}{|c|}{$K=t / 2 \pi$} & \multicolumn{1}{c|}{$K=3 t / 4 \pi$} \\
$p$ & $\left|Z-Z_{\text {approx }}\right|$ & $\left|Z-Z_{\text {approx }}\right|$ \\
\hline 1 & $4.290 \times 10^{-33}$ & $5.243 \times 10^{-44}$ \\
2 & $7.699 \times 10^{-24}$ & $1.708 \times 10^{-46}$ \\
3 & $3.273 \times 10^{-5}$ & $1.483 \times 10^{-18}$ \\
4 & $6.711 \times 10^{-2}$ & $4.964 \times 10^{-10}$ \\
5 & $5.970 \times 10^{-1}$ & $7.763 \times 10^{-7}$ \\
\hline
\end{tabular}

TABLE 5. Calculation of $Z(t)$ from (4.5) for different values of $p$ and $K$ when $t=100$. The number of terms in the finite sum is given by $M=\min \left\{M_{0}, 30\right\}$.

cost of increasing the effective cut-off point $n^{*} \sim K$ of the smoothed infinite sum. Secondly, although increasing $p$ results in a more rapid decay of this latter sum from the exponential factor $\exp \left[-(2 \pi n / t)^{2 p}\right]$, the overall attainable accuracy nevertheless is reduced on account of the decrease in the optimal truncation point of the finite series (see (4.4)) and the resultant reduction in the least term.

\section{Concluding remarks}

The expansion (2.8) involves a smoothing of the main sum (the original Dirichlet series) by the incomplete gamma function $Q\left(a,(n / K)^{2 p}\right)$ where $a$ is free to be chosen. The choice $a=1$ in $\S 4$ leads to the particularly simple smoothing by the exponential factor $\exp \left[-(n / K)^{2 p}\right]$. Another obvious choice is $a=\frac{1}{2}$ since $Q\left(\frac{1}{2}, z^{2}\right)=\operatorname{erfc}(z)$, and the main sum then is smoothed by the complementary error function. In this case with $p=1$ and $K=t / 2 \pi$, we have the expansion on the critical line $s=\frac{1}{2}+i t$

$$
\begin{gathered}
\zeta(s)=\sum_{n=1}^{\infty} n^{-s} \operatorname{erfc}(n / K)+\frac{\Gamma\left(1-\frac{1}{2} s\right)}{\sqrt{ } \pi} \frac{(t / 2 \pi)^{1-s}}{s-1} \\
+\frac{\chi(s)}{\sqrt{ } \pi} \sum_{m=0}^{M-1} \frac{(-)^{m}}{m !} \frac{t^{-2 m-1}}{m+\frac{1}{2}} A_{m}+R_{M}
\end{gathered}
$$

where

$$
A_{m}=(-)^{m-1} \cot \frac{1}{2} \pi s(1-s)_{2 m+1} \zeta(2+2 m-s)
$$

and

$$
\left|R_{M}\right|<\left(\frac{2}{\pi}\right)^{\frac{3}{2}} \frac{\zeta\left(2 M+\frac{1}{2}\right)}{M \Gamma\left(M+\frac{1}{2}\right)} C_{1}(M ; t) .
$$

In order for the finite sum to possess an asymptotic character, this formula again will require the choice $K \sim t / 2 \pi$, so that the resulting expansion is similarly of Gram type. Numerical results for the case $a=\frac{1}{2}$ and $p=1$ are shown in Table 4 .

The Berry-Keating formula, for which the leading term is given by (1.3), also involves the main sum smoothed by a complementary error function whose argument depends logarithmically on $n / N_{t}$. This difference in the $n$-dependence of the error function argument, however, results in the main sum cutting off after roughly $n^{*} \simeq N_{t} \simeq(t / 2 \pi)^{\frac{1}{2}}$ terms, so that their formula is of the more powerful RiemannSiegel type. The expansion (2.8) has been shown to produce a Gram-type expansion 
when $a=O(1)$, but it is a Riemann-Siegel-type expansion (see [8]) when $a=\frac{1}{2} s$. It would be of interest to explore the domain of $a$ values corresponding to the transition between these two categories of expansion.

Finally, the bound (4.6) on the remainder term $R_{M}$ was found numerically to be no longer realistic once $M \gtrsim\left[\frac{1}{2} t\right]$. We attribute this deterioration of $R_{M}$ to the relatively crude bounds employed in $\S 3$. It would be necessary to give a more refined analysis of the integral defining $R_{M}$ in (2.7) which possibly also takes cognizance of the oscillatory nature of the integrand. We remark, however, that if it were possible to assume the asymptotic nature of the expansion (4.2), then Table 2 indicates the enormous accuracy that could be achieved in the computation of $\zeta\left(\frac{1}{2}+i t\right)$ from (4.2) at optimal truncation.

Acknowledgement. One of the authors (S. C.) wishes to acknowledge the financial support of a Research Studentship at the University of Abertay Dundee.

\section{Appendix A. The relation between the expansions (1.4) and (2.2)}

We demonstrate how the result in (2.2) contains the expansion (1.4) as a special case. To see this, we let $a=\frac{1}{2} s$ and $p=1$ in (2.2) and (2.3) to find

$$
\zeta(s)=\sum_{n=1}^{\infty} n^{-s} Q\left(\frac{1}{2} s,(n / K)^{2}\right)+\frac{\Gamma\left(\frac{1}{2}\right)}{\Gamma\left(\frac{1}{2} s\right)} \frac{K^{1-s}}{s-1}+J
$$

where

$$
J=\frac{i}{2 \pi \Gamma\left(\frac{1}{2} s\right)} \int_{-c-\infty i}^{-c+\infty i} \Gamma\left(\frac{1}{2} s+\frac{1}{2} z\right) K^{z} \zeta(s+z) \frac{d z}{z}, \quad 0<c<\sigma, \quad|\arg K|<\pi / 4
$$

The above integral has only a single simple pole at $z=-s$ on the left of the path of integration, the remaining poles of the gamma function being cancelled by the trivial zeros of $\zeta(s+z)$ situated at $z=-s-2 k, k=1,2, \ldots$ Observing that $\zeta(0)=-\frac{1}{2}$, we therefore find, when $|\arg K|<\pi / 4$,

$$
\begin{aligned}
J & =-\frac{K^{-s}}{s \Gamma\left(\frac{1}{2} s\right)}+\frac{i}{2 \pi \Gamma\left(\frac{1}{2} s\right)} \int_{-c-\infty i}^{-c+\infty i} \Gamma\left(\frac{1}{2} s+\frac{1}{2} z\right) K^{z} \zeta(s+z) \frac{d z}{z} \quad(c>\sigma) \\
& =-\frac{K^{-s}}{s \Gamma\left(\frac{1}{2} s\right)}+\frac{\chi(s)}{\Gamma\left(\frac{1}{2}-\frac{1}{2} s\right)} \frac{1}{2 \pi i} \int_{c-\infty i}^{c+\infty i} \Gamma\left(\frac{1}{2}-\frac{1}{2} s+\frac{1}{2} z\right)(\pi K)^{-z} \zeta(1-s+z) \frac{d z}{z}
\end{aligned}
$$

upon use of the functional relation for $\zeta(s)$ and replacement of the variable $z$ by $-z$.

The integral in (A.3) is seen to be of the same form as that on the right-hand side of (2.1) (when $a=\frac{1}{2} s$ and $p=1$ ), with $s$ replaced by $1-s$ and the parameter $K$ by $(\pi K)^{-1}$. Hence, we find the result

$$
J=-\frac{K^{-s}}{s \Gamma\left(\frac{1}{2} s\right)}+\chi(s) \sum_{n=1}^{\infty} n^{s-1} Q\left(\frac{1}{2}-\frac{1}{2} s,(\pi n K)^{2}\right) .
$$

From (A.1), we then obtain the expansion

$$
\zeta(s)=\frac{K^{-s}}{\Gamma\left(\frac{1}{2} s\right)}\left(\frac{\sqrt{ } \pi K}{s-1}-\frac{1}{s}\right)+\sum_{n=1}^{\infty} n^{-s} Q\left(\frac{1}{2} s,(n / K)^{2}\right)
$$




$$
+\chi(s) \sum_{n=1}^{\infty} n^{s-1} Q\left(\frac{1}{2}-\frac{1}{2} s,(\pi n K)^{2}\right)
$$

valid for all values of $s(\neq 1)$ and $|\arg K|<\pi / 4$. Since $Q(a, z) \sim z^{a-1} e^{-z}$ as $|z| \rightarrow \infty$ in $|\arg z|<3 \pi / 2$, both the sums in (A.4) converge absolutely for $|\arg K| \leq \pi / 4$, so by analytic continuation, the expansion (A.4) holds in $|\arg K| \leq \pi / 4$. The choice $K=(\pi i)^{-\frac{1}{2}}$ and use of the conjugacy property $Q(\bar{a}, \bar{z})=\overline{Q(a, z)}$ together with $\chi\left(\frac{1}{2}+i t\right)=\exp (-2 i \vartheta)$, where $\vartheta$ is defined at (1.2), then yields the convergent expansion (1.4) on the critical line $s=\frac{1}{2}+i t$.

\section{Appendix B. The monotonicity of $F(x)$ and $G(x)$}

In this appendix, we establish two monotonicity properties connected with the function

$$
F(x)=\left|\frac{\Gamma\left(\alpha-\frac{1}{2} i x\right)}{\Gamma\left(\frac{1}{2}-\alpha+\frac{1}{2} i x\right)}\right|
$$

where $\alpha>0$ and $x \geq 0$. From [1, p. 256], we have

$$
F(x)=\frac{\Gamma(\alpha)}{\left|\Gamma\left(\frac{1}{2}-\alpha\right)\right|} \prod_{n=0}^{\infty}\left\{\frac{1+\frac{1}{4} x^{2} /\left(n+\frac{1}{2}-\alpha\right)^{2}}{1+\frac{1}{4} x^{2} /(n+\alpha)^{2}}\right\}^{\frac{1}{2}}
$$

which is evidently an even function of $x$. Logarithmic differentiation of (B.2) then yields

$$
\frac{F^{\prime}(x)}{F(x)}=\frac{1}{2}\left(\alpha-\frac{1}{4}\right) x \sum_{n=0}^{\infty} \frac{2 n+\frac{1}{2}}{\left[(n+\alpha)^{2}+\frac{1}{4} x^{2}\right]\left[\left(n+\frac{1}{2}-\alpha\right)^{2}+\frac{1}{4} x^{2}\right]},
$$

from which it seen that $F^{\prime}(x)>0$ for $x>0$, provided $\alpha>\frac{1}{4}$; we note that $F(x) \equiv 1$ when $\alpha=\frac{1}{4}$. Hence, $F(x)$ is a monotonically increasing function of $x$ when $\alpha>\frac{1}{4}$.

For $x \rightarrow+\infty$, the leading behavior of $F(x)$ is given by $F(x) \sim(x / 2)^{2 \alpha-\frac{1}{2}}$. To remove this growth, we define the scaled gamma function ratio by

$$
G(x)=\left(\frac{1}{2} x\right)^{\frac{1}{2}-2 \alpha} F(x)
$$

so that $G(x) \rightarrow 1$ as $x \rightarrow+\infty$ and, for $\alpha>\frac{1}{2}, G(x) \rightarrow+\infty$ as $x \rightarrow 0$. We now obtain the range of $\alpha$ values for which $G(x)$ is a monotonically decreasing function of $x$. From the standard asymptotic expansion of the ratio of two gamma functions $[1$, p.257] we obtain the behavior

$$
G(x)=1+\frac{4}{3} \alpha\left(\alpha-\frac{1}{4}\right)\left(\alpha-\frac{1}{2}\right) x^{-2}+O\left(x^{-4}\right), \quad x \rightarrow+\infty .
$$

Consequently, $G(x)$ is monotonically decreasing for large $x$ when $\alpha>\frac{1}{2}$; we note that $G(x)=\left(\tanh \frac{1}{2} \pi x\right)^{\frac{1}{2}}$ when $\alpha=\frac{1}{2}$.

Expressing the gamma function ratio in (B.1) in terms of a single gamma function, we have

$$
\begin{aligned}
G(x) & =\sqrt{\frac{2}{\pi}} x^{\frac{1}{2}-2 \alpha}\left|\Gamma(2 \alpha+i x) \sin \left\{\pi\left(\alpha+\frac{1}{2}\right)+\frac{1}{2} \pi i x\right\}\right| \\
& =\sqrt{\frac{2}{\pi}} \Gamma(2 \alpha) x^{\frac{1}{2}-2 \alpha} \prod_{n=0}^{\infty}\left\{1+\frac{x^{2}}{(n+2 \alpha)^{2}}\right\}^{-\frac{1}{2}}\left\{\sin ^{2} \pi\left(\alpha+\frac{1}{2}\right)+\sinh ^{2} \frac{1}{2} \pi x\right\}^{\frac{1}{2}},
\end{aligned}
$$


so that

$$
\begin{aligned}
\frac{G^{\prime}(x)}{G(x)}= & \frac{\frac{1}{2}-2 \alpha}{x}-\sum_{n=0}^{\infty} \frac{x}{(n+2 \alpha)^{2}+x^{2}} \\
& +\frac{1}{2} \pi \operatorname{coth} \frac{1}{2} \pi x\left\{1-\frac{\sin ^{2} \pi\left(\alpha+\frac{1}{2}\right)}{\sin ^{2} \pi\left(\alpha+\frac{1}{2}\right)+\sinh ^{2} \frac{1}{2} \pi x}\right\}
\end{aligned}
$$

Making use of the integral

$$
\frac{x}{x^{2}+\beta^{2}}=\int_{0}^{\infty} \sin x t e^{-\beta t} d t \quad(\beta>0)
$$

to express the sum appearing in (B.5) in the form

$$
\sum_{n=0}^{\infty} \frac{x}{(n+2 \alpha)^{2}+x^{2}}=\int_{0}^{\infty} \sin x t e^{-2 \alpha t} \frac{d t}{1-e^{-t}}
$$

and the identity

$$
\frac{1}{2} \pi \operatorname{coth} \frac{1}{2} \pi x=\frac{1}{x}+2 \int_{0}^{\infty} \frac{\sin x t}{e^{2 t}-1} d t
$$

we obtain from (B.5) the inequality

$$
\frac{G^{\prime}(x)}{G(x)} \leq \frac{\frac{3}{2}-2 \alpha}{x}-\int_{0}^{\infty} \sin x t H(t) d t
$$

where

$$
H(t)=\frac{e^{(1-2 \alpha) t}}{e^{t}-1}-\frac{2}{e^{2 t}-1} .
$$

Then, upon applying integration by parts twice and noting that $H(0)=\frac{3}{2}-2 \alpha$, we finally find the result

$$
\frac{G^{\prime}(x)}{G(x)} \leq \frac{1}{x^{2}} \int_{0}^{\infty} \sin x t H^{\prime \prime}(t) d t .
$$

It is easily demonstrated (we omit the details) that, for $\alpha \geq 1, H^{\prime \prime}(t)<0$ and $\left|H^{\prime \prime}(t)\right|$ is a monotonically decreasing function for $t \geq 0$. From the fact that (when $x>0) \int_{0}^{\infty} \sin x t f(t) d t>0$ for positive, monotonically decreasing functions $f(t)$, it follows from (B.6) that $G^{\prime}(x)<0$, and hence, that $G(x)$ is monotonically decreasing for $x>0$ when $\alpha \geq 1$. Direct numerical computation with Mathematica shows that $G(x)$ is in fact a monotonically decreasing function for $x>0$ when $\alpha>\alpha_{0}$ where $\alpha_{0} \approx 0.63605$.

\section{References}

1. M. Abramowitz and I. Stegun (Eds.), Handbook of Mathematical Functions, Dover, New York, 1965.

2. M. V. Berry and J. P. Keating, A new asymptotic representation for $\zeta\left(\frac{1}{2}+i t\right)$ and quantum spectral determinants, Proc. Roy. Soc. Lond. A437 (1992), 151-173.

3. H. M. Edwards, Riemann's Zeta Function, Academic Press, New York, 1974.

4. J. P. Gram, Note sur les zéros de la fonction $\zeta(s)$ de Riemann, Acta Mathematica 27 (1903), 289-304.

5. C. B. Haselgrove, Tables of the Riemann Zeta Function, Royal Society Mathematical Tables, Vol. 6, Cambridge University Press, 1963. 
6. R. B. Paris, An asymptotic representation for the Riemann zeta function on the critical line, Proc. Roy. Soc. Lond. A446 (1994), 565-587.

7. MACS 94:01, University of Abertay Dundee, 1994.

8. R. B. Paris and S. Cang, An asymptotic representation for $\zeta\left(\frac{1}{2}+i t\right)$, to appear in Methods Appl. Anal..

9. E. C. Titchmarsh, The Theory of Functions, Oxford University Press, 1939.

Division of Mathematical Sciences, University of Abertay Dundee, Dundee DD1 1HG, U.K. 